\title{
Observation of Potential Increase in the Central Cell due to ICRF Heating in the Non-Axisymmetric Anchor Cell on GAMMA 10/PDX ${ }^{*}$
}

\author{
Atsuto TANAKA, Makoto ICHIMURA, Mafumi HIRATA, Ryuya IKEZOE ${ }^{1)}$, Shuhei SUMIDA, \\ Seowon JANG, Koki IZUMI, Yushi KUBOTA, Ryo SEKINE, Hiroki KAYANO, \\ Mizuki SAKAMOTO and Yousuke NAKASHIMA \\ Plasma Research Center, University of Tsukuba, Tsukuba 305-8577, Japan \\ ${ }^{1)}$ Research Institute for Applied Mechanics, Kyushu University, Kasuga 816-8580, Japan
}

(Received 30 September 2018 / Accepted 21 December 2018)

\begin{abstract}
We used the additional Ion Cyclotron Range of Frequency (ICRF) heating in the anchor cell for the high density plasma production. When the ICRF waves are excited with antennas installed in the anchor cell, it is observed that the plasma potential increases significantly and forms non-axisymmetric potential distribution in the periphery of the central cell. Experiments with different frequencies were carried out to understand the physical mechanism of this potential change due to the ICRF waves. It is confirmed that the ion heating does not play an important role to the potential increase. The floating potentials on some tips set outside of the plasma in the anchor cell remarkably decrease during the additional heating and non-axisymmetric distribution of the floating potential in the central cell depends on the antenna location in the anchor cell. It is suggested that the radial transport of electrons near the antennas in the non-axisymmetric anchor cells with the ICRF antennas is a possible candidate of the potential increase in the central cell.
\end{abstract}

(c) 2019 The Japan Society of Plasma Science and Nuclear Fusion Research

Keywords: ICRF wave, anchor heating, potential, non-axisymmetric, GAMMA 10/PDX

DOI: $10.1585 /$ pfr. 14.2401028

\section{Introduction}

GAMMA 10/PDX is composed of multi magnetic mirror cells [1]. Plasmas are mainly produced and heated by using Ion Cyclotron Range of Frequency (ICRF) waves. The schematic drawings of the GAMMA 10/PDX device and magnetic field lines in the central and anchor cells are shown in Fig. 1. A central cell is the main plasma confinement region and plasmas in anchor cells keep the MHD stability of whole plasmas with the minimum-B configuration. Several ICRF antennas are installed on GAMMA 10/PDX. The locations and the shape of ICRF antennas are indicated in Fig. 1. Nagoya Type-III (Type-III) antennas installed at both end of the central cell are used for the plasma production and the ion heating in the anchor cells. Double Half Turn (DHT) antennas are also installed in the central cell for the ion heating there. In the anchor cells, Double Arc Type (DAT) antennas are installed for the additional anchor heating. Two DAT antennas (WAIDAT, WAO-DAT) are installed in the west anchor cell and one antenna (EAI-DAT) is installed in the east anchor cell as shown in Fig. 1. Recently, the divertor simulation experiments have been performed at the west end region by using end-loss plasmas from the central cell [2]. To ex-

author'se-mail: tanaka_atsuto@prc.tsukuba.ac.jp

*) This article is based on the presentation at the 12th International Conference on Open Magnetic Systems for Plasma Confinement (OS2018).

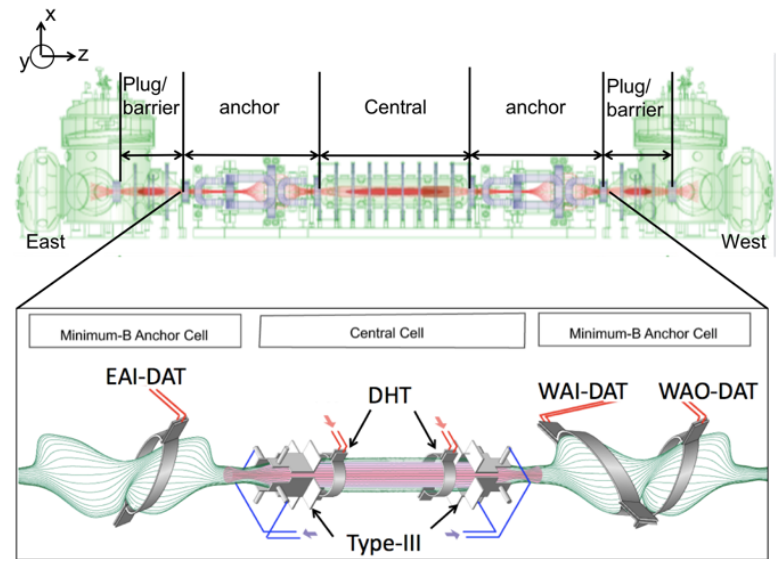

Fig. 1 The schematic drawings of the GAMMA 10/PDX device (above) and magnetic field lines in the axisymmetric central and non-axisymmetric anchor cells with several antenna (below).

tend the operation regime of the divertor simulation experiments, the additional anchor heating experiments have been carried out by using DAT antennas [3]. It has been observed that the significant increase of the density in the central and anchor cells and the end-loss ion flux to the end region. At the same time, it has been observed that the 
plasma potential in the central cell is affected by the additional anchor heating. Such a potential change by applying ICRF waves has been reported in other devices [4-6]. In these reports, the enhancement of the plasma potential has been observed in the central cell where ICRF antennas were installed. Ions in the central cell were confined and the reduction of end-loss ion flux was observed. On the other hand, the increase of the potential is observed in the central cell with the additional ICRF heating in the anchor cells and also the increase of the end-loss ion flux is observed in GAMMA 10/PDX. The purpose of this research is to understand the physical mechanism of this potential change due to the ICRF waves.

\section{Potential Measurements}

A metal limiter which is isolated electrically from the vacuum vessel is installed near the midplane of the central cell $(Z=33 \mathrm{~cm})$ [7]. The diameter of the limiter is $360 \mathrm{~mm}$. The limiter is divided into eight segments in the azimuthal direction. It is possible to measure the floating potential and its azimuthal distribution in the peripheral region of the central cell.

Gold Neutral Beam Probe (GNBP) is installed at $\mathrm{Z}=$ $118 \mathrm{~cm}$ in the central cell [8]. GNBP is a noble tool for the measurement of the core space potential.

\section{Experimental Results and Discus- sions}

In order to investigate the relationship between the additional anchor heating and the potential change, we applied ICRF waves with frequency of $6.0 \mathrm{MHz}, 9.9 \mathrm{MHz}$ and $16.26 \mathrm{MHz}$ by using the WAI-DAT antenna in the west anchor cell. Figure 2 shows an axial profile of the ion cyclotron frequency on GAMMA 10/PDX. Blue, red and green lines indicate the frequencies of $6.0 \mathrm{MHz}, 9.9 \mathrm{MHz}$ and $16.26 \mathrm{MHz}$, respectively. In the case of $6.0 \mathrm{MHz}$, there are no resonance layer in both the central cell and the west anchor cell. On the other hand, there are some cyclotron resonance layers in cases of $9.9 \mathrm{MHz}$ and $16.26 \mathrm{MHz}$.

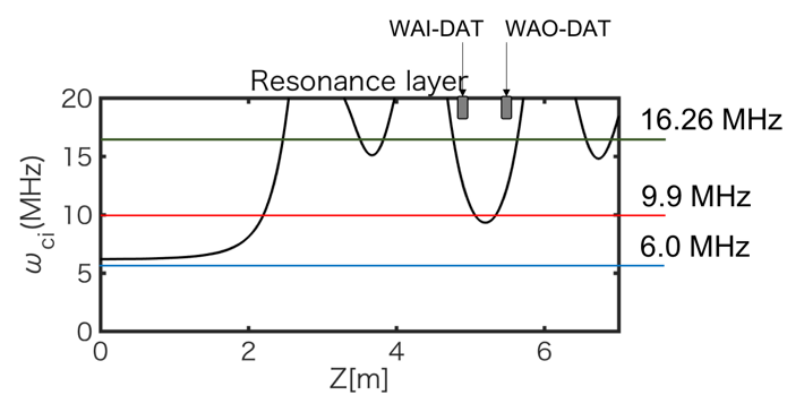

Fig. 2 Resonance layer of GAMMA 10/PDX (black line, $\mathrm{Z}=0$ to $8 \mathrm{~m}$ ) and various frequency of the additional anchor heating with WAI-DAT and WAO-DAT antenna.
Thus, we can evaluate about the effect of the ion heating on the potential change from experiments with these frequencies. The waves of $9.9 \mathrm{MHz}$ are often used for the additional anchor heating since the resonance layer of $9.9 \mathrm{MHz}$ is located near the midplane of the anchor cell. Because the electron temperature is quite low (several tens $\mathrm{eV}$ ) in the present experiment, the electron bounce frequency in the anchor cell is estimated to be lower than $10 \mathrm{MHz}$. The frequency of $16.26 \mathrm{MHz}$ is not expected to resonate the bounce electrons in non-axisymmetric anchor region. On the other hand, the frequency of $6.0 \mathrm{MHz}$ is expected to be a frequency that resonate the bounce electrons there. Therefore, we can evaluate about the effect of the bounce resonance of electrons by comparing the results of 6.0 $\mathrm{MHz}$ and 16.26 MHz.

The time evolution of the peripheral floating potential and the core space potential of the central cell are shown in Fig. 3. Plasmas are produced with east and west Type-III antennas from 54 to $240 \mathrm{~ms}$ at the frequencies of $9.9 \mathrm{MHz}$ and $10.3 \mathrm{MHz}$, respectively. The additional heating with the WAI-DAT antenna in the anchor cell is applied from 150 to $210 \mathrm{~ms}$ at a frequency of $6.0 \mathrm{MHz}$ or $9.9 \mathrm{MHz}$ or 16.26 MHz. As shown in Fig. 3, both the core space potential and the peripheral floating potential of the central cell are increased remarkably during the additional anchor heating. It was observed that the increase of the potential is independent of the applied frequencies. The similar tendencies of the potential changes in the potentials due to the additional anchor heating with the WAI-DAT antenna are also observed in the case with the WAO-DAT antenna or the EAI-DAT antenna.

(a)

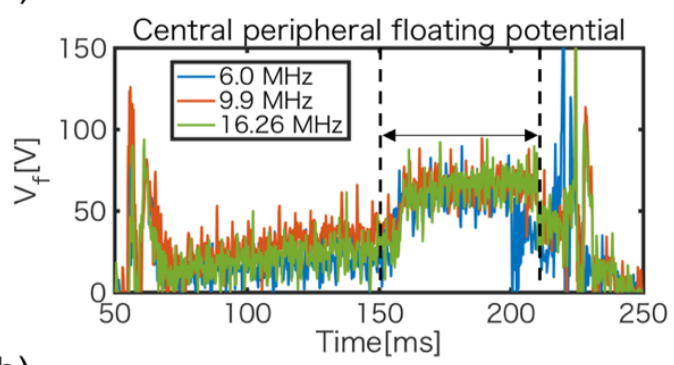

(b)

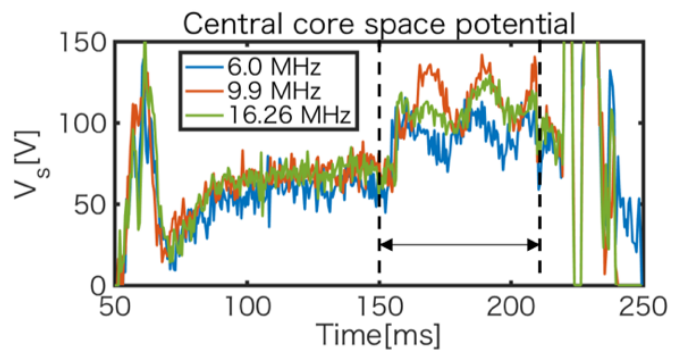

Fig. 3 The time evolution of (a) the peripheral floating potential (b) the core space potential, applying $9.9 \mathrm{MHz}$ for WAIDAT antenna during 150 to $210 \mathrm{~ms}$. 
(a)

Central peripheral floating potential

(b)
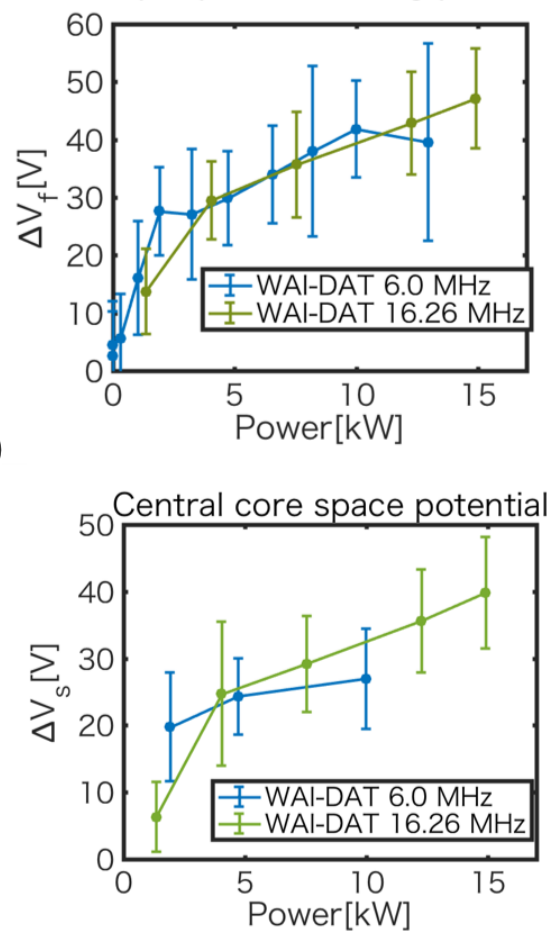

Fig. 4 Comparison of the power dependence of (a) the peripheral floating potential (b) the core space potential between applying $6.0 \mathrm{MHz}$ and $16.26 \mathrm{MHz}$.

Figure 4 shows the ICRF power dependences of the increase of (a) the peripheral floating potential and (b) the core space potential due to the additional anchor heating with $6.0 \mathrm{MHz}$ (blue line) and $16.26 \mathrm{MHz}$ (green line). Both potentials are increased with increase of the additional heating power and independent of the applied frequency. As shown in Fig. 4, the effect of the additional heating in the anchor cell to the core space potential can be estimated from the peripheral floating potential. It is clearly shown that the ion heating is not essential for the potential increase and the bounce resonance of electrons is not considered to be candidate of the potential increase since there are no frequency dependences.

Several metal tips are installed on the outside of plasmas in the anchor cell and can measure the floating potential. When the additional anchor heating is applied, the floating potentials on some of these tips decreases significantly. The time evolution of the floating potential in the anchor cell is shown in Fig. 5. Contrary to the floating potential in the central cell, a significant decrease of the floating potential is clearly observed in the anchor cell. In general, the potential is determined by the balance of ions and electrons. It is suggested that the radial loss of electrons occurs in the anchor cell with the non-axisymmetric configuration.

Azimuthal distributions of the floating potential mea-

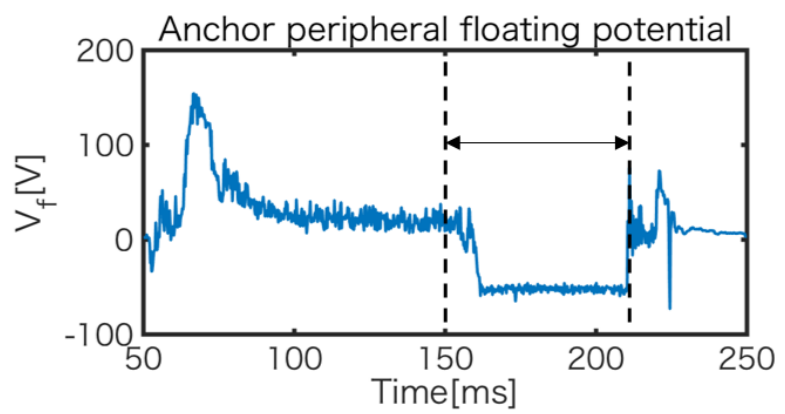

Fig. 5 The time evolution of an anchor peripheral floating potential, applying $6.0 \mathrm{MHz}$ for WAI-DAT antenna during 150 to $200 \mathrm{~ms}$.

(a)

Central floating potential distribution [V]

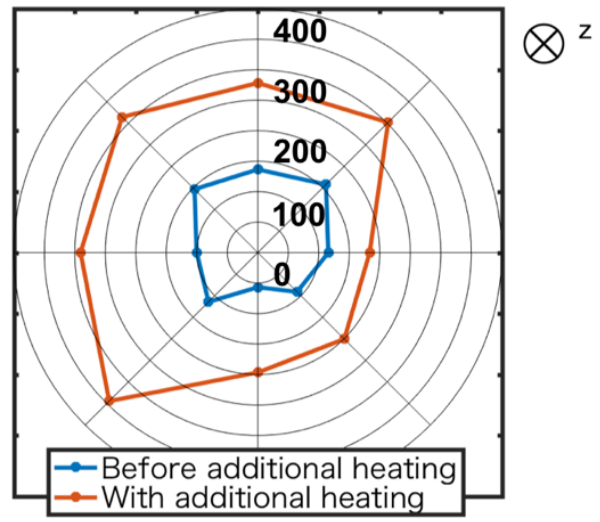

(b)

Central floating potential distribution [V]

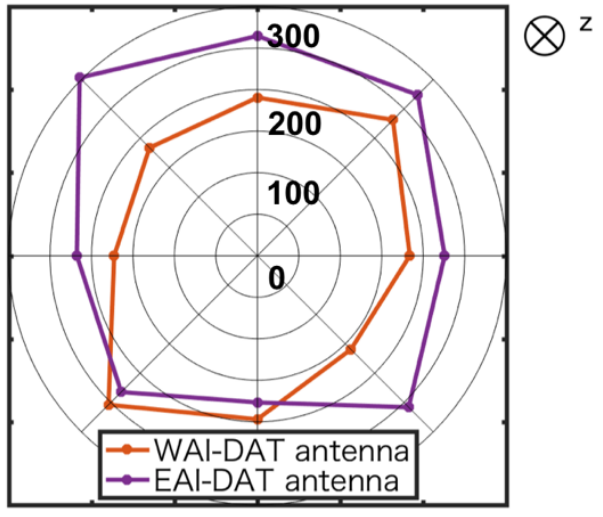

Fig. 6 Comparison of the floating potential distribution of (a) before and with additional anchor heating (b) difference antennas (WAI-DAT antenna and EAI-DAT antenna).

sured by the segmented limiter in the central cell are shown in Fig. 6. Figure 6(a) shows the floating potential distribution before (blue line) and during (red line) the additional anchor heating. The floating potentials increase and non-axisymmetry of their azimuthal distribution becomes 
stronger during the additional heating than before the additional heating. Figure 6(b) shows the azimuthal distributions of the limiter potential in the experiments with the anchor antennas of WAI-DAT (red line) and EAI-DAT (purple line). It is clearly shown in Fig. 6(b) that the direction of the non-axisymmetry is changed about 90 degrees with the WAI-DAT and the EAI-DAT antennas. Therefore, it is considered that the potential change strongly depends on the electromagnetic field near the non-axisymmetric anchor antennas. From Fig. 5 and Fig. 6, it is suggested that the radial transport of electrons in the non-axisymmetric anchor cells with the ICRF antennas is the possible candidate of the potential increase in the central cell.

\section{Summary}

It was observed that the additional anchor heating with ICRF antennas in the anchor cell enhances the increase of the plasma potential and forms the non-axisymmetric peripheral floating potential distribution in the central cell. Since the significant increase of the potential is observed with and without cyclotron resonance layers in the confinement region, the increase of the potential does not depend on the ion heating. The increase of the potential is also independent of the applied frequencies. Therefore, the bounce resonance of electrons will not play an important role to the potential increase. Since the negative floating potentials are observed during the additional anchor heating in the peripheral region of the anchor region and the azimuthal distribution of the floating potential in the central cell is influenced remarkably from the antenna location in the anchor cell with the non-axisymmetric configuration, it is suggested that the radial transport of electrons near the anchor antenna is a candidate of the potential increase.

\section{Acknowledgments}

The authors acknowledge the member of plasma research center in university Tsukuba for their collaboration. This work was partly supported by the bidirectional collaborative research program of the National Institute for Fusion Science, Japan (NIFS14KUGM086 and NIFS17KUGM132).

[1] M. Inutake et al., Phys. Rev. Lett. 55, 939 (1985).

[2] Y. Nakashima et al., Fusion Sci. Technol. 68, 28 (2015).

[3] Y. Saito et al., Trans. Fusion Sci. Technol. 63, 1T, 277 (2013).

[4] B.A. Nelson et al., Plasma Physics (1989-1993) 4, 3663 (1992).

[5] D.K. Smith et al., Phys. Fluids 29, 902 (1986).

[6] N. Hershkowitz et al., Phys. Rev. Lett. 55, 947 (1985).

[7] M. Hirata et al., Trans. Fusion Sci. Technol. 63, 1T, 247 (2013).

[8] M. Mizuguchi et al., Rev. Sci. Instrum. 79, 10 F309 (2008). 